\title{
Exit route evolved into entry path in plants
}

Chloroplast organelles in plant cells are thought to have evolved from bacterial cells. It emerges that the protein-import system in chloroplasts arose from components that export proteins out of bacteria. SEE LETTER P.125

\section{DANNY J. SCHNELL}

$\mathrm{C}$ hloroplasts are subcellular organelles that provide plants with abundant metabolic capabilities, most notably the ability to capture carbon from atmospheric carbon dioxide through the process of photosynthesis. It has been proposed ${ }^{1}$ that the plant kingdom began to evolve when a bacterium, similar to a present-day photosynthetic cyanobacterium, was engulfed by a host cell, and from this bacterial ancestor, chloroplasts eventually arose in the cellular descendants of the host cell. During chloroplast evolution, thousands of genes transferred from the intracellular bacterial genome to the host genome. However, these relocated genes encode proteins that need to be targeted to their site of function within the chloroplast. On page 125, Chen et al. ${ }^{2}$ report the identification of a component of the system that imports proteins into chloroplasts. Their finding illuminates how this evolved, and also provides mechanistic insight into how import is coordinated across the two membrane layers that form the chloroplast's outer envelope.

Most chloroplast proteins are made in the cytoplasm. They contain specific aminoacid sequences, termed transit peptides, that are used to direct these proteins from the cytoplasm, across the two membrane layers of the chloroplast and into the interior of the organelle $e^{3}$. Chen and colleagues' work addresses some key questions regarding this protein-import system. The first is, how do the multi-protein complexes, found at the outer and inner membranes of the chloroplast envelope (termed TOC and TIC, respectively) mediate transport in a coordinated way that prevents the mistargeting or misfolding of proteins as they transit through the intermembrane space? Under normal conditions, protein import across the outer and inner membranes seems to occur essentially simultaneously through the TOC and TIC complexes ${ }^{4}$. However, whether there is a physical connection between TOC and TIC, and if so, what its nature is, has been a mystery.

Chen $e t$ al. report the identification of a previously unknown component of the TIC complex, a protein that they name TIC236, which acts as a link between TIC and TOC. The authors discovered TIC236 using a biochemical approach to identify proteins that are associated with TOC components. TIC236 is anchored in the inner membrane, where it interacts with components of the TIC complex. Part of TIC236 extends into the intermembrane space, where it interacts with TOC75, a membrane protein that forms part of the channel in the TOC complex (Fig. 1a).

The authors report that, in the plant Arabidopsis thaliana, mutations that block the expression of TIC236 are lethal, and mutations that impair TIC236 function reduce the rates of protein import into chloroplasts compared to the import rate in wild-type $A$. thaliana. These results, in addition to the authors' studies of protein-protein interactions, provide compelling evidence that TIC236 provides a key physical link between TIC and TOC. Chen and a Plant cell

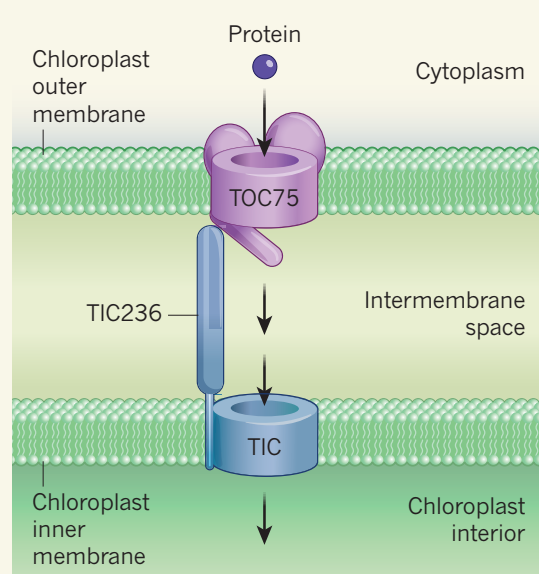

colleagues conducted a phylogenetic analysis that provides evidence for the co-evolution of the interacting domains of TOC75 and TIC236, supporting their hypothesis that these proteins evolved as components of interacting complexes. The ability to couple transit through TOC and TIC offers a way of ensuring efficient protein import. This is probably crucial during seedling development, when the rate of protein import into chloroplasts is high, and more than half of the total protein in a cell can be within the chloroplasts ${ }^{5}$.

The second key question this work addresses is, how did the TOC and TIC import systems evolve? Evidence for analogous systems that allow protein import into bacteria is lacking ${ }^{6}$, and, consequently, the evolutionary origin of the chloroplast protein-import system has been an open question. TOC75 is related to the OMP85 family of membrane proteins found on the outer membranes of chloroplasts, energygenerating organelles called mitochondria, and in Gram-negative bacteria, a group that includes the photosynthetic cyanobacteria ${ }^{7}$. Gram-negative bacteria have two membrane layers, and membrane proteins on their surface can be assembled and transported to the cell exterior with the help of protein complexes called TAM or BAM on the outer membrane of the cell. Membrane proteins in these complexes are members of the OMP85 family ${ }^{7}$. It has been proposed ${ }^{8}$ that TOC75 is derived from b Bacterium

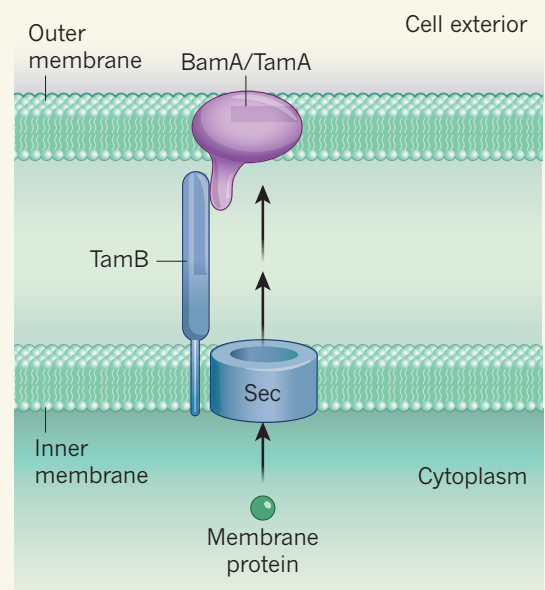

Figure 1 | Protein import into chloroplast organelles evolved from a bacterial protein-export system. a, Multi-protein complexes called TOC (purple) and TIC (blue), reside respectively on the outer and inner membrane layers of chloroplasts in plant cells. These complexes aid protein import (arrows) into the chloroplast. Chen et al. ${ }^{2}$ identified a protein component of TIC, which they termed TIC236, that directly associates with the protein TOC75 in the TOC complex of the plant Arabidopsis thaliana. This finding provides insight into how protein transit through both complexes is coordinated. $\mathbf{b}$, Bacterial proteins that are part of protein complexes called BAM or TAM aid protein export in the Gram-negative group of bacteria. The authors report that TIC236 is related to the bacterial protein TamB, and it has been proposed $^{8}$ that TOC75 is related to the bacterial proteins BamA or TamA. In Gram-negative bacteria, when membrane proteins are transported from the cytoplasm to the outer membrane, they cross the inner membrane through a protein complex called Sec. TamB is anchored in the inner membrane, and it aids the export of membrane proteins by facilitating transport between Sec and BamA or TamA proteins on the outer membrane. Chen and colleagues' results suggest that the protein-import system in chloroplasts evolved from this type of protein-export system in the microbial ancestor of chloroplasts. 
an ancestral protein related to components of the BAM or TAM systems in the ancestral bacterium that gave rise to the chloroplast.

Chen and colleagues demonstrate that TIC236 is related to TamB, which aids protein transport ${ }^{9}$ between bacterial membrane proteins that form a secretion system (called Sec), located on the inner membrane, and BAM or TAM components in the outer membrane (Fig. 1b). It therefore seems that a mechanism for coupling inner- and outer-membrane transport in chloroplasts has been evolutionarily conserved from an ancestral bacterial system.

However, despite this conservation, the chloroplast protein-import system has evolved to function in the reverse direction relative to the direction of transport in the bacterial export system ${ }^{5}$. The BAM and TAM complexes facilitate export of bacterial proteins from the cytoplasm to the outer membrane, whereas the TOC and TIC complexes import proteins from outside the chloroplast to inside it. This remarkable reversal of the direction of protein transport probably resulted from the gain of other TOC or TIC proteins that evolved from host-encoded genes to adapt the complexes for the purposes of protein import. These include TOC and TIC receptors and molecular motor proteins known to facilitate transport into the chloroplast ${ }^{3}$.

Chen and colleagues' results provide convincing evidence for the origin of key elements of the chloroplast protein-import system from an ancestral bacterial proteinexport system. Their insights also reveal the adaptation and consequent reversal of an existing protein-targeting pathway that was essential for the ancestral bacteria to successfully take up residence in a host cell, thereby enabling the host to take advantage of its guest's photosynthetic and metabolic capabilities.

Danny J. Schnell is in the Department of

Plant Biology, Michigan State University,

East Lansing, Michigan 48824, USA.

e-mail:schnelld@msu.edu

1. Archibald, J. M. Curr. Biol. 25, R911-R921 (2015).

2. Chen, Y.-L. et al. Nature 564, 125-129 (2018).

3. Paila, Y. D., Richardson, L. G. L. \& Schnell, D. J. J. Mol. Biol. 427, 1038-1060 (2015).

4. Chen, L. \& Li, H. Plant J. 92, 178-188 (2017).

5. Ellis, R. J. Trends Biochem. Sci. 4, 241-244 (1979).

6. Day, P. M. \& Theg, S. M. Photosynth. Res. 138, 315-326 (2018).

7. Heinz, E. \& Lithgow, T. Front. Microbiol. 5, 370 (2014).

8. Day, P. M., Potter, D. \& Inoue, K. Front. Plant Sci. 5, 535 (2014)

9. Heinz, E., Selkrig, J., Belousoff, M. J. \& Lithgow, T. Genome Biol. Evol. 7, 1628-1643 (2015).

This article was published online on 21 November 2018.

\section{Long-sought decay of the Higgs boson seen}

\begin{abstract}
Measurements of the strength of interactions between the Higgs boson and other particles test the current model of particle physics. A key part of this model has been confirmed by observing the most common decay of the Higgs boson.
\end{abstract}

\section{BORIS TUCHMING}

$\mathrm{I}$ n 2012, the famous Higgs boson was discovered by the ATLAS and CMS collaborations in proton-proton collisions at the Large Hadron Collider (LHC) at CERN near Geneva, Switzerland ${ }^{1,2}$. Now, writing in Physics Letters $B^{3}$ and Physical Review Letters ${ }^{4}$, the two collaborations report the observation of the Higgs boson decaying to a pair of elementary particles known as bottom quarks. This milestone in particle physics confirms the role of the Higgs field - the quantum field associated with the Higgs boson - in providing particles of matter with mass.

When the standard model of particle physics emerged in the 1960s, the main goal of the ad hoc Higgs field was to explain the masses of the weak vector bosons - the force carriers of the weak nuclear interaction. Mathematical consistency required the force carriers to be massless, whereas the extremely short range of the weak interaction was a signature of massive particles. The Higgs mechanism ${ }^{5-8}$ addressed this issue: the masses of the weak vector bosons are not intrinsic, but are the outcome of interactions between these particles and the all-pervasive Higgs field. It was quickly realized that elementary particles of matter from interactions with the Higgs field ${ }^{9,10}$. called fermions could also get their masses
Several decades later, twelve elementary fermions are known and are arranged in three families. The first family comprises three charged particles - the up quark, the down quark and the electron - and a neutral particle called the electron neutrino. These fermions are the basic ingredients of ordinary matter: the up and down quarks are the constituents of protons and neutrons, and electron neutrinos are emitted from certain radioactive decays.

For a reason that is not yet fully understood, two replicas of the first family exist. The second family consists of the charm quark, the strange quark, the muon and the muon neutrino, where the charged fermions have greater masses than their counterparts in the first family. And the third comprises the top quark, the bottom quark, the tau and the tau neutrino, where the charged fermions are even more massive.

After their discovery of the Higgs boson ${ }^{1,2}$, one objective of the ATLAS and CMS collaborations was to probe the particle's properties, such as its couplings to fermions - the strength of its interactions with fermions. In the current papers, the collaborations combined all the data that they recorded between 2011 and 2017, and each claims to have observed the decay of the Higgs boson to bottom quarks.

In both sets of data, the decay signal is larger than the background, which arises from other particle-physics processes. The statistical significance of the signal is 5.4 and 5.6 standard deviations for the ATLAS and CMS experiments, respectively - well above the conventional threshold of 5 standard deviations needed to claim observation. In addition, the overall yields of the decay are in agreement with standard-model predictions within an experimental uncertainty of roughly $20 \%$.

The Higgs boson decays almost immediately after it is produced. The probability that a particular decay will occur depends on the

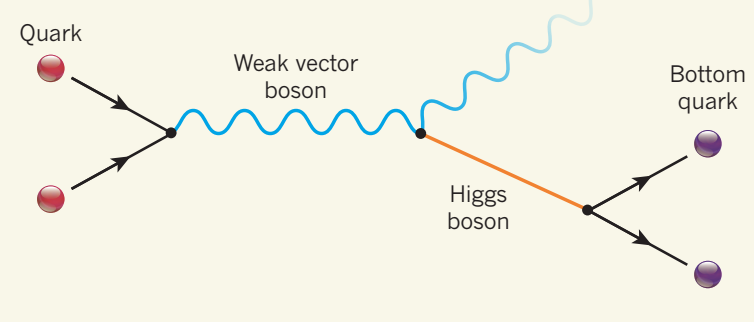

Figure 1 | Production of the Higgs boson together with a weak vector boson. The ATLAS ${ }^{3}$ and $\mathrm{CMS}^{4}$ collaborations report evidence that a particle known as the Higgs boson can decay to pairs of elementary particles called bottom quarks. To detect this decay, the collaborations looked for a particular process in which two quarks arising from colliding protons fuse to form a weak vector boson - a force carrier of the weak nuclear interaction. The weak vector boson emits a Higgs boson that decays to bottom quarks. 